Article

\title{
Existence and Approximate Controllability of Mild Solutions for Fractional Evolution Systems of Sobolev-Type
}

\author{
Yue Liang
}

check for updates

Citation: Liang, Y. Existence and Approximate Controllability of Mild Solutions for Fractional Evolution Systems of Sobolev-Type. Fractal Fract. 2022, 6, 56. https://doi.org/ $10.3390 /$ fractalfract6020056

Academic Editor: Rodica Luca

Received: 5 January 2022

Accepted: 18 January 2022

Published: 22 January 2022

Publisher's Note: MDPI stays neutral with regard to jurisdictional claims in published maps and institutional affiliations.

Copyright: (C) 2022 by the author. Licensee MDPI, Basel, Switzerland. This article is an open access article distributed under the terms and conditions of the Creative Commons Attribution (CC BY) license (https:// creativecommons.org/licenses/by/ $4.0 /)$.
Center for Quantitative Biology, College of Science, Gansu Agricultural University, Lanzhou 730070, China; liangy@gsau.edu.cn; Tel.: +86-139-1915-6640

\begin{abstract}
This paper investigates the existence and approximate controllability of Riemann-Liouville fractional evolution systems of Sobolev-type in abstract spaces. At first, a group of sufficient conditions is established for the existence of mild solutions without the compactness of operator semigroup. Then the approximate controllability is studied under the assumption that the corresponding linear system is approximate controllability. The proof is based on the fixed point theory and the method of operator semigroup. An example is given as an application of the obtained results.
\end{abstract}

Keywords: fractional evolution systems; approximate controllability; Sobolev operator; compactness; Schauder fixed point theorem

MSC: 26A33; 93B05

\section{Introduction}

Let $X$ be a Hilbert space, whose norm is denoted by $\|\cdot\|$. We consider the fractional evolution equation of sobolev type with the Riemann-Liouville derivative of the form

$$
\left\{\begin{array}{l}
{ }^{L} D_{t}^{\alpha}(E x(t))=A x(t)+f(t, x(t))+B u(t), \quad t \in J^{\prime}:=(0, b], \\
\left.I_{t}^{1-\alpha}(E x(t))\right|_{t=0}+g(x)=x_{0},
\end{array}\right.
$$

where ${ }^{L} D_{t}^{\alpha}$ is the Riemann-Liouville fractional derivative operator of order $\alpha \in(0,1), I_{t}^{1-\alpha}$ is the fractional integral operator of order $1-\alpha, A: D(A) \subset X \rightarrow X$ and $E: D(E) \subset X \rightarrow$ $X$ are linear operators, $B$ is a linear bounded operator from $U$ to $X$; here $U$ is another Hilbert space, the control function $u \in L^{p}(J, U)$ for $p \alpha>1, x_{0} \in X, f$ is the nonlinear function and $g$ represents the nonlocal function which satisfies specific conditions.

Fractional differential equations, including of the Caputo type and Riemann-Liouville type, have been proved to be crucial tools in portraying the hereditary and memory property of various materials and processes. In 2011, Du et al. [1] pointed out that RiemannLiouville fractional derivatives are more suitable to describe certain characteristics of viscoelastic materials than Caputo ones. Therefore, it is significant to study RiemannLiouville fractional differential systems. In 2013, Zhou et al. [2], applying the Laplace transform technique and probability density functions, presented a suitable concept of mild solutions of Riemann-Liouville fractional evolution equations, and proved the existence of mild solutions for the fractional Cauchy problems under the cases that the $C_{0}$-semigroup is compact or noncompact. For the existence of mild solutions of fractional evolution equations, we refer to [3-8] and the references therein. In these papers, the compactness of operator semigroup or the measure of non-compactness conditions on nonlinearity are required. Sometimes, in order to obtain the uniqueness of mild solutions, the Lipschitz condition is also assumed.

In recent years, the controllability of fractional evolution equations has gained considerable attention. Generally speaking, the controllability of fractional evolution equations in 
abstract spaces includes two cases: the exact controllability and the approximate controllability. When we study the exact controllability of fractional evolution systems in abstract spaces, we assume that the control operator has a bounded inverse operator in a quotient space. However, if the state space is infinite dimensional and the operator semigroup is compact, the inverse of the control operator may not exist, see [9]. Hence, the assumptions for the exact controllability are too strong. Contrasting with the exact controllability, approximate controllability is more suitable to describe the natural phenomena. There are many research works focusing on the approximate controllability of fractional evolution systems, see [10-12] and the references therein. In [10], Chang et al. investigated the approximate controllability of fractional differential systems of Sobolev type in Banach spaces under the assumption that the resolvent operators, generated by the linear part, are compact. Sakthivel et al. [11] studied the approximate controllability of nonlinear fractional stochastic evolution systems when the linear part generates a compact semigroup. Recently, In [12], Yang demonstrated the existence and approximate controllability of mild solutions for $\alpha \in(1,2)$-order fractional evolution equations of Sobolev type when the pair $(A, E)$ generates a compact resolvent family.

Inspired by the above mentioned papers, the aim of this work is to investigate the existence and approximate controllability of Riemann-Liouville fractional evolution system (1) in Hilbert space $X$. By using the Schauder fixed point theorem and the operator semigroup theory, we first prove the existence of mild solutions of the considered system without the compactness of operator semigroup and the measure of non-compactness conditions on nonlinearity. Then the approximate controllability is studied under the assumption that the corresponding linear system is approximate controllability. It is emphasized that the compactness of the operator semigroup and the Lipschitz continuity of nonlinearity are deleted in our work. The redundant assumptions on the linear operator $E$, such as the conditions $\left[C_{1}\right]$ and $\left[C_{4}\right]$ of [13], are removed in this paper.

\section{Preliminaries}

Let $J=[0, b]$ and $C(J, X)$ be the continuous function space. Denote by

$$
C_{1-\alpha}(J, X):=\left\{x: \cdot{ }^{1-\alpha} x(\cdot) \in C(J, X)\right\} .
$$

Then $C_{1-\alpha}(J, X)$ is a Banach space endowed with the norm $\|x\|_{C_{1-\alpha}}=\sup _{t \in J} t^{1-\alpha}\|x(t)\|$.

At first, for any $h \in L^{p}(J, X)$ with $p \alpha>1$, we consider the following linear fractional initial value problem

$$
\left\{\begin{array}{l}
{ }^{L} D_{t}^{\alpha}(E x(t))=A x(t)+h(t), \quad t \in J^{\prime}, \\
\left.I_{t}^{1-\alpha}(E x(t))\right|_{t=0}+g(x)=x_{0} .
\end{array}\right.
$$

Throughout this paper, we suppose the following assumptions on $A$ and $E$.

$(A 1)$ The linear operator $A$ is densely defined and closed.

(A2) $D(E) \subset D(A)$ and $E$ is bijective.

(A3) The linear operator $E^{-1}: X \rightarrow D(E) \subset X$ is compact.

By $(A 1)-(A 3)$, the linear operator $A E^{-1}: X \rightarrow X$ is bounded due to the closed graph theorem. Hence, $A E^{-1}$ generates a $C_{0}$-semigroup $T(t)(t \geq 0)$, which is expressed by $T(t)=e^{A E^{-1} t}$ for $t \geq 0$. We suppose that $M:=\sup _{t \geq 0}\|T(t)\|<+\infty$.

Remark 1. Contrasting with [13], we delete the redundant conditions $\left[C_{1}\right]$ and $\left[C_{4}\right]$ of $[13]$ in our paper. Hence, the results obtained in this work extends the results of [13].

Applying the Riemann-Liouville fractional integral operator on both sides of (2), we obtain 


$$
\begin{aligned}
E x(t) & =\left.\frac{t^{\alpha-1}}{\Gamma(\alpha)} I_{t}^{1-\alpha}(E x(t))\right|_{t=0}+I_{t}^{\alpha} A x(t)+I_{t}^{\alpha} h(t) \\
& =\frac{t^{\alpha-1}}{\Gamma(\alpha)}\left[x_{0}-g(x)\right]+\frac{1}{\Gamma(\alpha)} \int_{0}^{t}(t-s)^{\alpha-1}[A x(s)+h(s)] d s .
\end{aligned}
$$

Let $\lambda>0$. Taking the Laplace transform

$$
\widehat{x}(\lambda)=\int_{0}^{\infty} e^{-\lambda t} x(t) d t
$$

and

$$
\widehat{h}(\lambda)=\int_{0}^{\infty} e^{-\lambda t} h(t) d t
$$

on both sides of the above equality, we can obtain

$$
\begin{aligned}
E \widehat{x}(t) & =\frac{1}{\lambda^{\alpha}}\left[x_{0}-g(x)\right]+\frac{1}{\lambda^{\alpha}} A E^{-1} E \widehat{x}(\lambda)+\frac{1}{\lambda^{\alpha}} \widehat{h}(\lambda) \\
& =\left(\lambda^{\alpha} I-A E^{-1}\right)^{-1}\left[x_{0}-g(x)\right]+\left(\lambda^{\alpha} I-A E^{-1}\right)^{-1} \widehat{h}(\lambda) \\
& =\int_{0}^{\infty} e^{-\lambda^{\alpha} s} T(s)\left[x_{0}-g(x)\right] d s+\int_{0}^{\infty} e^{-\lambda^{\alpha} s} T(s) \widehat{h}(\lambda) d s,
\end{aligned}
$$

where $\left(\lambda^{\alpha} I-A E^{-1}\right)^{-1}=\int_{0}^{\infty} e^{-\lambda^{\alpha} s} T(s) d s$. Consider the one-side stable probability density function

$$
\xi_{\alpha}(\theta)=\frac{1}{\pi} \sum_{n=1}^{\infty}(-1)^{n-1} \theta^{-\alpha n-1} \frac{\Gamma(n \alpha+1)}{n !} \sin (n \pi \alpha), \theta \in(0,+\infty),
$$

whose Laplace transform is given by

$$
\int_{0}^{\infty} e^{-\lambda \theta} \xi_{\alpha}(\theta) d \theta=e^{-\lambda^{\alpha}}, \quad \alpha \in(0,1)
$$

A similar argument as in [2] shows that

$$
\begin{aligned}
E \widehat{x}(\lambda)= & \int_{0}^{\infty} e^{-\lambda t} \int_{0}^{\infty} \alpha \theta \xi_{\alpha}(\theta) T\left(t^{\alpha} \theta\right) t^{\alpha-1}\left[x_{0}-g(x)\right] d \theta d t \\
& +\int_{0}^{\infty} e^{-\lambda t} \int_{0}^{t} \int_{0}^{\infty} \alpha \theta \xi_{\alpha}(\theta) T\left((t-s)^{\alpha} \theta\right)(t-s)^{\alpha-1} h(s) d \theta d s d t,
\end{aligned}
$$

where $\xi_{\alpha}(\theta)=\frac{1}{\alpha} \theta^{-1-\frac{1}{\alpha}} \mathcal{\omega}_{\alpha}\left(\theta^{-\frac{1}{\alpha}}\right)$. This fact implies that

$$
\begin{aligned}
E x(t)= & \int_{0}^{\infty} \alpha \theta \xi_{\alpha}(\theta) T\left(t^{\alpha} \theta\right) t^{\alpha-1}\left[x_{0}-g(x)\right] d \theta \\
& +\int_{0}^{t} \int_{0}^{\infty} \alpha \theta \xi_{\alpha}(\theta) T\left((t-s)^{\alpha} \theta\right)(t-s)^{\alpha-1} h(s) d \theta d s .
\end{aligned}
$$

Thus, we obtain

$$
x(t)=t^{\alpha-1} T_{E}(t)\left[x_{0}-g(x)\right]+\int_{0}^{t}(t-s)^{\alpha-1} T_{E}(t-s) h(s) d s,
$$

where

$$
T_{E}(t)=E^{-1} \int_{0}^{\infty} \alpha \theta \xi_{\alpha}(\theta) T\left(t^{\alpha} \theta\right) d \theta
$$

Remark 2. When $E=I, I: X \rightarrow X$ is the identity operator, we have

$$
T_{I}(t)=\int_{0}^{\infty} \alpha \theta \xi_{\alpha}(\theta) T\left(t^{\alpha} \theta\right) d \theta, t \geq 0 .
$$


Therefore, $T_{E}(t)=E^{-1} T_{I}(t)$ for all $t \geq 0$.

From the above arguments, we introduce the definition of mild solution of the system (1) as follows.

Definition 1. For each $u \in L^{p}(J, U), p \alpha>1$, a function $x \in C_{1-\alpha}(J, X)$ is called a mild solution of the system (1) if $\left.I_{t}^{1-\alpha}(E x(t))\right|_{t=0}+g(x)=x_{0}$ and

$$
x(t)=t^{\alpha-1} T_{E}(t)\left[x_{0}-g(x)\right]+\int_{0}^{t}(t-s)^{\alpha-1} T_{E}(t-s)[f(s, x(s))+B u(s)] d s, t \in J^{\prime} .
$$

For the operator family $\left\{T_{E}(t)\right\}_{t \geq 0}$, we have the following lemma.

Lemma 1. Let the assumptions $(A 1)-(A 3)$ hold. Then $\left\{T_{E}(t)\right\}_{t \geq 0}$ has the following properties:

(i) For fixed $t \geq 0, T_{E}(t)$ is a linear and bounded operator, i.e., for any $x \in X$,

$$
\left\|T_{E}(t) x\right\| \leq \frac{M\left\|E^{-1}\right\|}{\Gamma(\alpha)}\|x\| .
$$

(ii) $\left\{T_{E}(t)\right\}_{t \geq 0}$ is continuous in the uniform operator topology for $t \geq 0$.

(iii) $\left\{T_{E}(t)\right\}_{t \geq 0}$ is compact.

Proof. From Proposition 3.1 of [2] and Remark 2, it is easy to verify that $(i)$ holds. By virtue of the definition of the operator $T(t)(t \geq 0)$ and the Lebesgue dominated convergence theorem, we can deduce (ii). Next, we prove (iii). For any $r>0, x \in X$ with $\|x\| \leq r$, we have

$$
\begin{aligned}
\left\|T_{I}(t) x\right\| & \leq \alpha M \int_{0}^{\infty} \theta \xi_{\alpha}(\theta) d \theta\|x\| \\
& \leq \frac{\alpha M}{\Gamma(\alpha+1)}\|x\| \\
& \leq \frac{M r}{\Gamma(\alpha)}
\end{aligned}
$$

This fact means that $T_{I}(t)$ maps bounded subset of $X$ into the bounded set. Then $E^{-1} T_{I}(t)$ maps the bounded subset of $X$ into relatively compact set due to the compactness of $E^{-1}$. Thus, $\left\{T_{E}(t)\right\}_{t \geq 0}$ is compact.

Definition 2. Let $K_{f}(b)=\left\{x(b): x\right.$ be a mild solution of the system $(1)$ for some $\left.u \in L^{p}(J, U)\right\}$. If $\overline{K_{f}(b)}=X$, the system (1) is said to be approximate controllability on $J$.

We consider the linear fractional control system corresponding to (1) in the form

$$
\left\{\begin{array}{l}
{ }^{L} D_{t}^{\alpha}(E x(t))=A x(t)+B u(t), \quad t \in J^{\prime}, \\
I_{t}^{1-\alpha}(E x(t))=x_{0} .
\end{array}\right.
$$

Define two operators $\Pi_{0}^{b}$ and $R\left(\epsilon, \Pi_{0}^{b}\right)$ by

$$
\begin{gathered}
\Pi_{0}^{b}=\int_{0}^{b}(b-s)^{\alpha-1} T_{E}(b-s) B B^{*} T_{E}^{*}(b-s) d s, \\
R\left(\epsilon, \Pi_{0}^{b}\right)=\left(\epsilon I+\Pi_{0}^{b}\right)^{-1}, \quad \epsilon>0,
\end{gathered}
$$

where $B^{*}$ and $T_{E}^{*}(t)$ denote the adjoint operators of $B$ and $T_{E}(t)$, respectively. Then, $\Pi_{0}^{b}$ is a linear operator. From [14], we obtain the following result. 
Lemma 2. The following conditions are equivalent:

(i) The linear fractional control system (4) is approximately controllable on J.

(ii) The operator $\Pi_{0}^{b}$ is positive, that is, $\left\langle x^{*}, \Pi_{0}^{b} x^{*}\right\rangle>0$ for all nonzero $x^{*} \in X^{*}$.

(iii) For any $x \in X,\left\|\epsilon R\left(\epsilon, \Pi_{0}^{b}\right) x\right\| \rightarrow 0$ as $\epsilon \rightarrow 0^{+}$.

\section{Existence and Approximate Controllability}

In order to study the approximate controllability of the fractional control system (1), we first investigate the existence of solutions for the following integral system

$$
\left\{\begin{array}{l}
x(t)=t^{\alpha-1} T_{E}(t)\left[x_{0}-g(x)\right]+\int_{0}^{t}(t-s)^{\alpha-1} T_{E}(t-s)[f(s, x(s))+B u(s ; x)] d s, \quad t \in J^{\prime}, \\
u(t ; x)=B^{*} T_{E}^{*}(b-t) R\left(\epsilon, \Pi_{0}^{b}\right) \mathcal{P}(x), \\
\mathcal{P}(x)=x_{b}-b^{\alpha-1} T_{E}(b)\left(x_{0}-g(x)\right)-\int_{0}^{b}(b-s)^{\alpha-1} T_{E}(b-s) f(s, x(s)) d s,
\end{array}\right.
$$

where $x_{b}$ is an arbitrary element in $X$ which is different from $x_{0}$. By Definition 1 , the mild solution of the system (1) is equivalent to the solution of the integral system (5) for $u(\cdot ; x) \in$ $L^{p}(J, X)$.

For this purpose, we make the following assumptions.

(A4) $f: J \times X \rightarrow X$ satisfies the following conditions.

(i) For each $x \in X, f(\cdot, x): J \rightarrow X$ is strongly measurable, and for every $t \in J, f(\cdot, x)$ : $X \rightarrow X$ is continuous.

(ii) For any $r>0$, there is a function $\phi \in L^{p}\left(J, \mathbb{R}^{+}\right), p \alpha>1$ such that

$$
\|f(t, x)\| \leq \phi(t)
$$

for any $t \in J$ and $x \in X$ with $\|x\| \leq r$.

$(A 5) g: C_{1-\alpha}(J, X) \rightarrow X$ is continuous and maps bounded subset of $C_{1-\alpha}(J, X)$ into the bounded set.

(A6) $B: U \rightarrow X$ is a bounded linear operator, i.e., $\exists M_{B}>0$ such that $\|B\| \leq M_{B}$.

(A7) $\left\|R\left(\epsilon, \Pi_{0}^{b}\right)\right\| \leq \frac{1}{\epsilon}$ for all $\epsilon>0$.

For any $r>0$, let $B_{r}=\left\{x \in C_{1-\alpha}(J, X):\|x\|_{C_{1-\alpha}} \leq r\right\}$. Then $B_{r}$ is a nonempty bounded, closed and convex subset of $C_{1-\alpha}(J, X)$. By the assumption $(A 5)$ we know that there exists a constant $M_{1}>0$ such that $\|g(x)\| \leq M_{1}$ for any $x \in B_{r}$. From the assumption (A6) we deduce that $B u \in L^{p}(J, X)$ for any $u \in L^{p}(J, X)$ with $p \alpha>1$.

Lemma 3. For any $\mathcal{F} \in L^{p}(J, X)$, the operator $\aleph: L^{p}(J, X) \rightarrow C(J, X)$, defined by

$$
(\aleph \mathcal{F})(\cdot)=\cdot{ }^{1-\alpha} \int_{0}^{\cdot}(\cdot-s)^{\alpha-1} T_{E}(\cdot-s) \mathcal{F}(s) d s,
$$

is compact.

Proof. Denote by

$$
\left(\aleph_{0} \mathcal{F}\right)(t)=t^{1-\alpha} \int_{0}^{t}(t-s)^{\alpha-1} T_{I}(t-s) \mathcal{F}(s) d s
$$

It follows from Lemma 1 that

$$
\left\|\left(\aleph_{0} \mathcal{F}\right)(t)\right\| \leq \frac{M}{\Gamma(\alpha)}\left(\frac{b p-b}{p \alpha-1}\right)^{1-\frac{1}{p}}\|\mathcal{F}\|_{L^{p}} .
$$

So, owing to the compactness of $E^{-1}$, we conclude that the set

$$
\left\{(\aleph \mathcal{F})(t)=E^{-1}\left(\aleph_{0} \mathcal{F}\right)(t): \mathcal{F} \in L^{p}(J, X), t \in J\right\}
$$

is relatively compact in $X$. 
Next, we will prove that the set $\left\{\aleph \mathcal{F}: \mathcal{F} \in L^{p}(J, X)\right\}$ is equi-continuous in $C(J, X)$. For $t_{1}, t_{2} \in J$ with $0 \leq t_{1}<t_{2}<b$, we have

$$
\begin{aligned}
\left\|(\aleph \mathcal{F})\left(t_{2}\right)-(\aleph \mathcal{F})\left(t_{1}\right)\right\| & \leq\left\|\left(t_{2}^{1-\alpha}-t_{1}^{1-\alpha}\right) \int_{0}^{t_{2}}\left(t_{2}-s\right)^{\alpha-1} T_{E}\left(t_{2}-s\right) \mathcal{F}(s) d s\right\| \\
& +t_{1}^{1-\alpha}\left\|\int_{0}^{t_{1}}\left[\left(t_{2}-s\right)^{\alpha-1}-\left(t_{1}-s\right)^{\alpha-1}\right] T_{E}\left(t_{2}-s\right) \mathcal{F}(s) d s\right\| \\
& +t_{1}^{1-\alpha}\left\|\int_{0}^{t_{1}}\left(t_{1}-s\right)^{\alpha-1}\left[T_{E}\left(t_{2}-s\right)-T_{E}\left(t_{1}-s\right)\right] \mathcal{F}(s) d s\right\| \\
& +t_{1}^{1-\alpha}\left\|\int_{t_{1}}^{t_{2}}\left(t_{2}-s\right)^{\alpha-1} T_{E}\left(t_{2}-s\right) \mathcal{F}(s) d s\right\| \\
& =\sum_{i=1}^{4} I_{i} .
\end{aligned}
$$

Obviously, if $t_{2}-t_{1} \rightarrow 0$, we have

$$
\begin{aligned}
& I_{1}=\left\|\left(t_{2}^{1-\alpha}-t_{1}^{1-\alpha}\right) \int_{0}^{t_{2}}\left(t_{2}-s\right)^{\alpha-1} T_{E}\left(t_{2}-s\right) \mathcal{F}(s) d s\right\| \\
& \leq \frac{M\left\|E^{-1}\right\|}{\Gamma(\alpha)}\left(\frac{p-1}{p \alpha-1}\right)^{1-\frac{1}{p}}\|\mathcal{F}\|_{L^{p}}\left(t_{2}-t_{1}\right)^{1-\alpha} \\
& \rightarrow 0, \\
& I_{2} \quad=t_{1}^{1-\alpha}\left\|\int_{0}^{t_{1}}\left[\left(t_{2}-s\right)^{\alpha-1}-\left(t_{1}-s\right)^{\alpha-1}\right] T_{E}\left(t_{2}-s\right) \mathcal{F}(s) d s\right\| \\
& \quad \leq \frac{M\left\|E^{-1}\right\| b^{1-\alpha}}{\Gamma(\alpha)} \int_{0}^{t_{1}}\left[\left(t_{2}-s\right)^{\alpha-1}-\left(t_{1}-s\right)^{\alpha-1}\right] \mathcal{F}(s) d s \\
& \quad \rightarrow 0
\end{aligned}
$$

and

$$
\begin{aligned}
I_{4} & =t_{1}^{1-\alpha}\left\|\int_{t_{1}}^{t_{2}}\left(t_{2}-s\right)^{\alpha-1} T_{E}\left(t_{2}-s\right) \mathcal{F}(s) d s\right\| \\
& \leq \frac{M\left\|E^{-1}\right\| b^{1-\alpha}}{\Gamma(\alpha)}\left(\frac{p-1}{p \alpha-1}\right)^{1-\frac{1}{p}}\|\mathcal{F}\|_{L^{p}}\left(t_{2}-t_{1}\right)^{\frac{p \alpha-1}{p}} \\
& \rightarrow 0 .
\end{aligned}
$$

Since $T_{E}(t)$ is continuous in the uniform operator topology for $t \geq 0$, we obtain that

$$
\begin{aligned}
I_{3} & =t_{1}^{1-\alpha}\left\|\int_{0}^{t_{1}}\left(t_{1}-s\right)^{\alpha-1}\left[T_{E}\left(t_{2}-s\right)-T_{E}\left(t_{1}-s\right)\right] \mathcal{F}(s) d s\right\| \\
& \leq \sup _{s \in\left[0, t_{1}\right]}\left\|T_{E}\left(t_{2}-s\right)-T_{E}\left(t_{1}-s\right)\right\|\left(\frac{b p-b}{p \alpha-1}\right)^{1-\frac{1}{p}}\|\mathcal{F}\|_{L^{p}} \\
& \rightarrow 0
\end{aligned}
$$

as $t_{2}-t_{1} \rightarrow 0$. Consequently, we have

$$
\left\|(\aleph \mathcal{F})\left(t_{2}\right)-(\aleph \mathcal{F})\left(t_{1}\right)\right\| \rightarrow 0\left(t_{2}-t_{1} \rightarrow 0\right) .
$$

This fact yields that the set $\left\{\aleph \mathcal{F}: \mathcal{F} \in L^{p}(J, X)\right\}$ is equi-continuous in $C(J, X)$. According to the Ascoli-Arzela theorem, the set $\left\{\aleph \mathcal{F}: \mathcal{F} \in L^{p}(J, X)\right\}$ is relatively compact in $C(J, X)$. 
Theorem 1. Let the assumptions $(A 1)-(A 7)$ hold. Then, the system (1) has at least one mild solution on $\mathrm{J}$.

Proof. For any $\epsilon>0$, let $r>0$ be large enough such that

$$
\begin{gathered}
r \geq N^{*}\left\|x_{b}\right\|+\frac{M\left\|E^{-1}\right\|}{\Gamma(\alpha)}\left(N^{*} b^{\alpha-1}+1\right)\left(\left\|x_{0}\right\|+M_{1}\right)+\frac{M\left\|E^{-1}\right\|}{\Gamma(\alpha)}\left(\frac{b p-b}{p \alpha-1}\right)^{1-\frac{1}{p}}\left(\|\phi\|_{L^{p}}\left(N^{*}+1\right),\right. \\
\text { where } N^{*}=\frac{b}{\epsilon}\left(\frac{M M_{B}\left\|E^{-1}\right\|}{\Gamma(\alpha)}\right)^{2}\left(\frac{p-1}{p \alpha-1}\right)^{1-\frac{1}{p}} . \text { Define an operator } \Phi: B_{r} \rightarrow C_{1-\alpha}(J, X) \text { by } \\
(\Phi x)(t)=t^{\alpha-1} T_{E}(t)\left[x_{0}-g(x)\right]+\int_{0}^{t}(t-s)^{\alpha-1} T_{E}(t-s)[f(s, x(s))+B u(s ; x)] d s,
\end{gathered}
$$

where

$$
\begin{gathered}
u(s ; x)=B^{*} T_{E}^{*}(b-s) R\left(\epsilon, \Pi_{0}^{b}\right) \mathcal{P}(x), \\
\mathcal{P}(x)=x_{b}-b^{\alpha-1} T_{E}(b)\left(x_{0}-g(x)\right)-\int_{0}^{b}(b-s)^{\alpha-1} T_{E}(b-s) f(s, x(s)) d s .
\end{gathered}
$$

Step 1. We will prove $\Phi: B_{r} \rightarrow B_{r}$.

For any $\epsilon>0$, by assumptions $(A 4)-(A 7)$ and Lemma 1 , we have

$$
\|u(t ; x)\| \leq \frac{M M_{B}\left\|E^{-1}\right\|}{\epsilon \Gamma(\alpha)}\|\mathcal{P}(x)\|, \quad x \in B_{r}, \quad t \in J^{\prime}
$$

and

$$
\|\mathcal{P}(x)\| \leq\left\|x_{b}\right\|+\frac{M\left\|E^{-1}\right\| b^{\alpha-1}}{\Gamma(\alpha)}\left(M_{1}+\left\|x_{0}\right\|\right)+\frac{M\left\|E^{-1}\right\|}{\Gamma(\alpha)}\left(\frac{b p-b}{p \alpha-1}\right)^{1-\frac{1}{p}}\|\phi\|_{L^{p}}, \quad x \in B_{r} .
$$

Together this fact with (6), for any $\epsilon>0$, we have

$$
\begin{aligned}
t^{1-\alpha}\|(\Phi x)(t)\| & \leq\left\|T_{E}(t)\left[x_{0}-g(x)\right]\right\|+t^{1-\alpha}\left\|\int_{0}^{t}(t-s)^{\alpha-1} T_{E}(t-s)[f(s, x(s))+B u(s)] d s\right\| \\
& \leq \frac{M\left\|E^{-1}\right\|}{\Gamma(\alpha)}\left(\left\|x_{0}\right\|+M_{1}\right)+b^{1-\alpha} \frac{M\left\|E^{-1}\right\|}{\Gamma(\alpha)} \int_{0}^{t}(t-s)^{\alpha-1}\left(\phi(s)+M_{B}\|u(s)\|\right) d s \\
& \leq \frac{M\left\|E^{-1}\right\|}{\Gamma(\alpha)}\left(\left\|x_{0}\right\|+M_{1}\right)+\frac{M\left\|E^{-1}\right\|}{\Gamma(\alpha)}\left(\frac{b p-b}{p \alpha-1}\right)^{1-\frac{1}{p}}\left(\|\phi\|_{L^{p}}+M_{B}\|u\|_{L^{p}}\right) \\
\leq & N^{*}\left\|x_{b}\right\|+\frac{M\left\|E^{-1}\right\|}{\Gamma(\alpha)}\left(N^{*} b^{\alpha-1}+1\right)\left(\left\|x_{0}\right\|+M_{1}\right) \\
& +\frac{M\left\|E^{-1}\right\|}{\Gamma(\alpha)}\left(\frac{b p-b}{p \alpha-1}\right)^{1-\frac{1}{p}}\|\phi\|_{L^{p}}\left(N^{*}+1\right) \\
\leq & r .
\end{aligned}
$$

Thus, $\|\Phi x\|_{C_{1-\alpha}}=\sup _{t \in J} t^{1-\alpha}\|(\Phi x)(t)\| \leq r$, which implies $\Phi: B_{r} \rightarrow B_{r}$.

Step 2. $\Phi: B_{r} \rightarrow B_{r}$ is continuous.

Let $\left\{x_{n}\right\} \subset B_{r}$ with $x_{n} \rightarrow \bar{x}$ as $n \rightarrow \infty$. From the continuity of $f$ and $g$, we have

$$
f\left(t, x_{n}(t)\right) \rightarrow f(t, \bar{x}(t)), \quad t \in J
$$

and

$$
g\left(x_{n}\right) \rightarrow g(\bar{x})
$$

as $n \rightarrow \infty$. Since

$$
\left\|(t-s)^{\alpha-1}\left[f\left(s, x_{n}(s)\right)-f(s, \bar{x}(s))\right]\right\| \leq 2(t-s)^{\alpha-1} \phi(s) \in L^{1}\left(J, \mathbb{R}^{+}\right),
$$


it follows from the Lebesgue dominated convergence theorem that

$$
\begin{aligned}
& t^{1-\alpha}\left\|\left(\Phi x_{n}\right)(t)-(\Phi \bar{x})(t)\right\| \\
\leq & \left\|T_{E}(t)\left(g\left(x_{n}\right)-g(\bar{x})\right)\right\|+b^{1-\alpha} \frac{M\left\|E^{-1}\right\|}{\Gamma(\alpha)} \int_{0}^{t}(t-s)^{\alpha-1}\left\|f\left(s, x_{n}(s)\right)-f(s, \bar{x}(s))\right\| d s \\
\rightarrow & 0(n \rightarrow \infty) .
\end{aligned}
$$

Hence,

$$
\left\|\Phi x_{n}-\Phi \bar{x}\right\|_{C_{1-\alpha}} \rightarrow 0
$$

as $n \rightarrow \infty$ and $\Phi: B_{r} \rightarrow B_{r}$ is continuous.

Step 3. The set $\left\{\Phi x: x \in B_{r}\right\}$ is relatively compact in $C_{1-\alpha}(J, X)$.

In order to prove the relative compactness of $\left\{\Phi x: x \in B_{r}\right\}$ in $C_{1-\alpha}(J, X)$, we prove that the set $\left\{{ }^{1-\alpha} \Phi x(\cdot): x \in B_{r}\right\}$ is relatively compact in $C(J, X)$.

Denote by

$$
\left(\Phi_{1} x\right)(t)=T_{E}(t)\left(x_{0}-g(x)\right), \quad t \in J
$$

and

$$
\left(\Phi_{2} x\right)(t)=t^{1-\alpha} \int_{0}^{t}(t-s)^{\alpha-1} T_{E}(t-s)[f(s, x(s))+B u(s)] d s, \quad t \in J .
$$

Then for any $t \in J$, we have

$$
t^{1-\alpha} \Phi x(t)=\left(\Phi_{1} x\right)(t)+\left(\Phi_{2} x\right)(t)
$$

It is sufficient to prove that $\left\{\Phi_{1} x: x \in B_{r}\right\}$ and $\left\{\Phi_{2} x: x \in B_{r}\right\}$ are relatively compact in $C(J, X)$.

For any $x \in B_{r}$ and $t \in J$, by virtue of

$$
\left\|T_{I}(t)\left(x_{0}-g(x)\right)\right\| \leq \frac{M}{\Gamma(\alpha)}\left(\left\|x_{0}\right\|+M_{1}\right),
$$

we obtain that $\left\{\left(\Phi_{1} x\right)(t): x \in B_{r}, t \in J\right\}$ is relatively compact in $X$ owing to the compactness of $E^{-1}$. It is obvious that the set $\left\{\Phi_{1} x: x \in B_{r}\right\}$ is equi-continuous in $C(J, X)$ because $T_{E}(t)$ is continuous in the uniform operator topology for $t \geq 0$. Hence, it follows from the Ascoli-Arzela theorem that the set $\left\{\Phi_{1} x: x \in B_{r}\right\}$ is relatively compact in $C(J, X)$.

By assumptions $(A 4)$ and $(A 6)$, we know that

$$
f(t, x(t))+B u(t) \in L^{p}(J, X) .
$$

By Lemma 3, the set $\left\{\Phi_{2} x: x \in B_{r}\right\}$ is relatively compact in $C(J, X)$. Consequently, the set $\left\{\Phi x: x \in B_{r}\right\}$ is relatively compact in $C_{1-\alpha}(J, X)$.

Hence, $\Phi$ is completely continuous in $C_{1-\alpha}(J, X)$. By the Schauder fixed point theorem, $\Phi$ has at least one fixed point in $B_{r}$, which is the mild solution of the system (1).

Remark 3. In [15], Lian et al. proved the existence of mild solutions of fractional evolution equations under the assumption that the nonlocal function $g$ is continuous, uniformly bounded and satisfies some other conditions. In [2], Zhou et al. investigated the existence of mild solutions of fractional evolution equations when the nonlocal function $g$ is Lipschitz continuous or completely continuous. In our Theorem 1, we only assume that the nonlocal function $g$ is continuous and maps bounded subset into bounded set, without the Lipschitz continuity and the complete continuity and any other extra conditions we obtain the existence of mild solutions of the fractional evolution Equation (1). Hence, Theorem 1 greatly extends the main results in [2,15].

If the assumptions $(A 4)$ and $(A 5)$ are replaced by the following conditions: $(A 4)^{\prime} f: J \times X \rightarrow X$ satisfies the following conditions. 
(i) For each $x \in X, f(\cdot, x): J \rightarrow X$ is strongly measurable, and for every $t \in J, f(\cdot, x):$ $X \rightarrow X$ is continuous.

(ii) There exists a function $\psi \in L^{p}\left(J, \mathbb{R}^{+}\right), p \alpha>1$ and a constant $\rho>0$ such that

$$
\|f(t, x)\| \leq \psi(t)+\rho t^{1-\alpha}\|x\|, \quad t \in J, \quad x \in X .
$$

$(A 5)^{\prime} g: C_{1-\alpha}(J, X) \rightarrow X$ is continuous and there exists a constant $M_{2}>0$ such that $\|g(x)\| \leq M_{2}$ for any $x \in C_{1-\alpha}(J, X)$.

then by Theorem 1 we can obtain the following existence theorem.

Theorem 2. Let the assumptions $(A 1)-(A 3),(A 4)^{\prime},(A 5)^{\prime},(A 6)$ and $(A 7)$ hold. Then the system (1) has at least one mild solution in $C_{1-\alpha}(J, X)$.

Proof. It is clear that $(A 5)^{\prime} \Rightarrow(A 5)$ and $(A 4)^{\prime} \Rightarrow(A 4)$ with $\phi(\cdot)=\psi(\cdot)+r \rho^{1-\alpha} \in$ $L^{p}(J, X)$ for any $r>0$ and $x \in B_{r}$. Therefore, by Theorem 1 we can prove that the system (1) has a mild solution $x \in C_{1-\alpha}(J, X)$.

Now, we state and prove the approximate controllability of the fractional control system (1).

Theorem 3. Let the conditions $(A 1)-(A 3),(A 4)^{\prime \prime},(A 5)^{\prime}$ and $(A 6)$ be satisfied, where $(A 4)^{\prime \prime} f: J \times X \rightarrow X$ satisfies the following conditions.

(i) For each $x \in X, f(\cdot, x): J \rightarrow X$ is strongly measurable, and for every $t \in J, f(\cdot, x)$ : $X \rightarrow X$ is continuous.

(ii) There exist a function $\varphi \in L^{p}\left(J, \mathbb{R}^{+}\right)$with $p \alpha>1$ such that

$$
\|f(t, x)\| \leq \varphi(t), \quad \forall t \in J, \quad x \in X
$$

In addition, the linear fractional control system (4) is approximately controllable on J. Then the fractional control system (1) is approximately controllable on J.

Proof. It is clear that $(A 4)^{\prime \prime} \Rightarrow(A 4)$ and $(A 5)^{\prime} \Rightarrow(A 5)$. By Lemma 2 we know that the condition (H7) holds. It follows from Theorem 1 that the system (1) has a mild solution $x_{\epsilon} \in C_{1-\alpha}(J, X)$ for every $\epsilon>0$, which is expressed by

$$
\begin{aligned}
x_{\epsilon}(t) & =t^{\alpha-1} T_{E}(t)\left[x_{0}-g(x)\right]+\int_{0}^{t}(t-s)^{\alpha-1} T_{E}(t-s) f\left(s, x_{\epsilon}(s)\right) d s \\
& +\int_{0}^{t}(t-s)^{\alpha-1} T_{E}(t-s) B B^{*} T_{E}^{*}(b-s) R\left(\epsilon, \Pi_{0}^{b}\right)\left[x_{b}-b^{\alpha-1} T_{E}(b)\left(x_{0}-g(x)\right)\right. \\
& \left.-\int_{0}^{b}(b-\theta)^{\alpha-1} T_{E}(b-\theta) f\left(\theta, x_{\epsilon}(\theta)\right) d \theta\right] d s .
\end{aligned}
$$

In view of $I-\Pi_{0}^{b}\left(\epsilon I+\Pi_{0}^{b}\right)^{-1}=\epsilon R\left(\epsilon, \Pi_{0}^{b}\right)$, we have

$$
x_{\epsilon}(b)=x_{b}-\epsilon R\left(\epsilon, \Pi_{0}^{b}\right) p\left(x_{\epsilon}\right),
$$

where

$$
p\left(x_{\epsilon}\right)=x_{b}-b^{\alpha-1} T_{E}(b)\left(x_{0}-g\left(x_{\epsilon}\right)\right)-\int_{0}^{b}(b-s)^{\alpha-1} T_{E}(b-s) f\left(s, x_{\epsilon}(s)\right) d s .
$$

By the assumption $(A 5)^{\prime}$, we have

$$
\left\|b^{\alpha-1}\left(x_{0}-g\left(x_{\epsilon}\right)\right)\right\| \leq b^{\alpha-1}\left(\left\|x_{0}\right\|+M_{2}\right) .
$$


Then the set $\left\{b^{\alpha-1} T_{E}(b)\left(x_{0}-g\left(x_{\epsilon}\right)\right)\right\}$ is relatively compact since $T_{E}(b)$ is a compact operator. There exists a subsequence of $\left\{b^{\alpha-1} T_{E}(b)\left(x_{0}-g\left(x_{\epsilon}\right)\right)\right\}$, still denoted by itself, and a function $g^{*}$ such that

$$
b^{\alpha-1} T_{E}(b)\left(x_{0}-g\left(x_{\epsilon}\right)\right) \rightarrow g^{*} \quad\left(\epsilon \rightarrow 0^{+}\right) .
$$

By means of $(A 4)^{\prime \prime}$ we have

$$
\left\|f\left(\cdot, x_{\epsilon}(\cdot)\right)\right\|_{L^{p}}=\left(\int_{0}^{b}\left\|f\left(s, x_{\epsilon}(s)\right)\right\|^{p} d s\right)^{\frac{1}{p}} \leq\|\varphi\|_{L^{p}} .
$$

Hence, the set $\left\{f\left(\cdot, x_{\epsilon}(\cdot)\right)\right\}$ is bounded in $L^{p}(J, X)$. So there is a subsequence, still denoted by $\left\{f\left(\cdot, x_{\epsilon}(\cdot)\right)\right\}$, converges weakly to some $f^{*}(\cdot) \in L^{p}(J, X)$, that is,

$$
f\left(s, x_{\epsilon}(s)\right) \stackrel{w}{\longrightarrow} f^{*}(s), \text { a.e. } s \in J
$$

as $\epsilon \rightarrow 0$. By Lemma 3 and the Lebesgue dominated convergence theorem, we can obtain

$$
\int_{0}^{b}(b-s)^{\alpha-1} T_{E}(b-s) f\left(s, x_{\epsilon}(s)\right) d s \rightarrow \int_{0}^{b}(b-s)^{\alpha-1} T_{E}(b-s) f^{*}(s) d s
$$

as $\epsilon \rightarrow 0$. Denote by

$$
h=x_{b}-g^{*}-\int_{0}^{b}(b-s)^{\alpha-1} T_{E}(b-s) f^{*}(s) d s .
$$

Then by the definition of $p\left(x_{\epsilon}\right)$, we obtain that

$$
p\left(x_{\epsilon}\right) \rightarrow h(\epsilon \rightarrow 0) .
$$

Consequently, we have

$$
\begin{aligned}
\left\|x_{\epsilon}(b)-x_{b}\right\| & =\left\|\epsilon R\left(\epsilon, \Pi_{0}^{b}\right) p\left(x_{\epsilon}\right)\right\| \\
& =\left\|\epsilon R\left(\epsilon, \Pi_{0}^{b}\right)\left(p\left(x_{\epsilon}\right)-h\right)\right\|+\mid \epsilon R\left(\epsilon, \Pi_{0}^{b}\right) h \| \\
& \rightarrow 0 \quad(\epsilon \rightarrow 0) .
\end{aligned}
$$

By Definition 2, the fractional control system (1) is approximately controllable on $J$.

\section{An Example}

Consider the Sobolev-type partial differential equation with Riemann-Liouville fractional derivatives

$$
\left\{\begin{array}{l}
{ }^{L} D_{t}^{\frac{3}{4}}\left[\left(I-\frac{\partial^{2}}{\partial y^{2}}\right) x(t, y)\right]=\frac{\partial^{2}}{\partial y^{2}} x(t, y)+\frac{e^{-3 t} \sqrt{\sin x(t, y)}}{3+|x(t, y)|}+u(t), \quad(t, y) \in(0,1] \times[0, \pi], \\
x(t, 0)=x(t, \pi)=0, \quad t \in[0,1] \\
\left.I_{0^{+}}^{1-\alpha}\left[\left(I-\frac{\partial^{2}}{\partial y^{2}}\right) x(t, y)\right]\right|_{t=0}+\sum_{i=1}^{m} c_{i} \sqrt[3]{\sin \left(t^{1-\alpha} x(t, y)\right)+7}=x_{0}(y)
\end{array}\right.
$$

where $c_{i}>0, i=1,2, \cdots, m$ are given positive constants.

Let $X=U:=L^{2}[0, \pi]$. Denote $D(A)=D(E):=\left\{x \in X: x, x^{\prime}\right.$ are absolutely continuous, $x^{\prime \prime} \in X$ and $\left.x(t, 0)=x(t, \pi)=0\right\}$. We define two operators $A: D(A) \subset X \rightarrow$ $X$ and $E: D(E) \subset X \rightarrow X$ by

$$
A x=\frac{\partial^{2}}{\partial y^{2}} x, \quad x \in D(A) ; \quad E x=\left(I-\frac{\partial^{2}}{\partial y^{2}}\right) x, \quad x \in D(E) .
$$


Let $e_{n}(y)=\sqrt{\frac{2}{\pi}} \sin n y, n \in \mathbb{N}$ be the orthonormal set of eigenvectors of $A$. By $[4,16]$, we have

$$
A x=-\Sigma_{n=1}^{\infty} n^{2}\left\langle x, e_{n}\right\rangle e_{n}, \quad x \in D(A)
$$

and

$$
E x=\Sigma_{n=1}^{\infty}\left(1+n^{2}\right)\left\langle x, e_{n}\right\rangle e_{n}, \quad x \in D(E) .
$$

This implies, for any $x \in H$, that

$$
\begin{aligned}
& E^{-1} x=\sum_{n=1}^{\infty} \frac{1}{1+n^{2}}\left\langle x, e_{n}\right\rangle e_{n} \\
& A E^{-1} x=\sum_{n=1}^{\infty} \frac{-n^{2}}{1+n^{2}}\left\langle x, e_{n}\right\rangle e_{n}
\end{aligned}
$$

and

$$
T(t) x=\sum_{n=1}^{\infty} e^{\frac{-n^{2}}{1+n^{2}} t}\left\langle x, e_{n}\right\rangle e_{n}
$$

where $T(t) x=e^{A E^{-1} t} x, t \geq 0$. Then $E^{-1}$ is a linear operator which is compact and $\left\|E^{-1}\right\| \leq 1$. Hence,

$$
T_{E}(t)=\frac{3}{4} \int_{0}^{\infty} E^{-1} \theta \xi_{\frac{3}{4}}(\theta) T\left(t^{\frac{3}{4}} \theta\right) d \theta
$$

with

$$
\left\|T_{E}(t) x\right\| \leq \frac{1}{\Gamma\left(\frac{3}{4}\right)}\|x\|
$$

where

$$
\xi_{\frac{3}{4}}(\theta)=\frac{1}{\pi} \sum_{n=1}^{\infty}(-1)^{n-1} \theta^{-\frac{3}{4} n-1} \frac{\Gamma\left(\frac{3}{4} n+1\right)}{n !} \sin \left(\frac{3}{4} n \pi\right), \theta \in(0,+\infty) .
$$

Let $x(t)(y)=x(t, y)$. Denote

$$
f(t, x(t))(y)=\frac{e^{-3 t} \sqrt{\sin x(t, y)}}{3+|x(t, y)|}
$$

and

$$
g(x)(y)=\sum_{i=1}^{m} c_{i} \sqrt[3]{\sin \left(t^{1-\alpha} x(t, y)\right)+7}
$$

Then the problem (7) can be rewritten as the abstract control system (1). Moreover, the assumptions $(A 1)-(A 6)$ are fulfilled with $\|f(t, x)\|_{X}=\frac{1}{3}$ and $\|g(x)\|_{X} \leq 2 \sum_{i=1}^{m} c_{i}$. If the linear system corresponding to (7) is approximately controllable on $[0,1]$, then by Theorem 3 , the fractional partial differential equation of $(7)$ is approximately controllable on $[0,1]$.

\section{Conclusions}

In this paper, with the aid of the compactness of the operator $E^{-1}$, we prove the existence of mild solutions of the fractional evolution system (1) without the compactness of operator semigroup. The Lipschitz continuity and the compactness of the nonlocal function $g$ are not needed in our main results. Under the assumption that the associate linear control system (4) is approximately controllable, the approximate controllability of the fractional evolution system (1) is also studied.

Funding: The research is supported by the National Natural Science Function of China (No. 11701457).

Institutional Review Board Statement: Not applicable.

Informed Consent Statement: Not applicable.

Data Availability Statement: Not applicable. 
Conflicts of Interest: The author declares no conflict of interest.

\section{References}

1. Du, M.L.; Wang, Z.H. Initialized fractional differential equations with Riemann-Liouville fractional-order derivative. Eur. Phys. J. Spec. Top. 2011, 193, 49-60. [CrossRef]

2. Zhou, Y.; Zhang, L.; Shen, X.H. Existence of mild solutions for fractional evolution equations. J. Int. Equ. Appl. $2013,25,557-585$. [CrossRef]

3. Benchaabane, A.; Sakthivel, R. Sobolev-type fractional stochastic differential equations with non-Lipschitz coefficients. J. Comput. Appl. Math. 2017, 312, 65-73. [CrossRef]

4. Fečkan, M.; Wang, J.R.; Zhou, Y. Controllability of fractional functional evolution equations of Sobolev type via characteristic solution operators. J. Optim. Theory Appl. 2013, 156, 79-95.

5. Liang, J.; Yang, H. Controllability of fractional integro-differential evolution equations with nonlocal conditions. Appl. Math. Comput. 2015, 254, 20-29. [CrossRef]

6. Shu, X.B.; Wang, Q.Q. The existence and uniqueness of mild solutions for fractional differential equations with nonlocal conditions of order $1<\alpha<2$. Comput. Math. Appl. 2012, 64, 2100-2110.

7. Yang, H. Existence results of mild solutions for the fractional stochastic evolution equations of Sobolev type. Symmetry 2020, 12, 1031. [CrossRef]

8. Yang, H.; Zhao, Y.J. Controllability of fractional evolution systems of Sobolev type via resolvent operators. Bound. Value Prob. 2020, 2020, 119. [CrossRef]

9. Hernández, E.; O’Regan, D. Controllability of Volterra-Fredholm type systmes in Banach spaces. J. Frankl. Inst. 2009, 346, 95-101. [CrossRef]

10. Chang, Y.K.; Pereira, A.; Ponce, R. Approximate controllability for fractional differential equations of Sobolev type via properties on resolvent operators. Fract. Calc. Appl. Anal. 2017, 20, 963-987. [CrossRef]

11. Sakthivel, R.; Suganya, S.; Anthoni, S.M. Approximate controllability of fractional stochastic evolution equations. Comput. Math. Appl. 2012, 63, 660-668. [CrossRef]

12. Yang, H. Approximate controllability of Sobolev type fractional evolutin equations of order $\alpha \in(1,2)$ via resolvent operator. $J$. Appl. Anal. Comput. 2021, 11, 2981-3000.

13. Balachandran, K.; Dauer, J.P. Controllability of functional differential systems of Sobolev type in Banach spaces. Kybernetika 1998, 34, 349-357.

14. Mahmudov, N.I. Approximate controllability of semilinear deterministic and stochastic evolution equation in abstract spaces. SIAM J. Control Optim. 2003, 42, 1604-1622. [CrossRef]

15. Lian, T.T.; Fan, Z.B.; Li, G. Approximate controllability of semilinear fractional differential systems of order $1<q<2$ via resolvent operators. Filomat 2017, 18, 5769-5781.

16. Lightbourne, J.H.; Rankin, S.M. A partial functional differential equation of Sobolev type. J. Math. Anal. Appl. 1983, 93, 328-337. [CrossRef] 